\title{
ONE STEP INTEGRATION OF GOLD COATED SENSORS WITH OSTE POLYMER CARTRIDGES BY LOW TEMPERATURE DRY BONDING
}

\author{
N. Sandström, R. Z. Shafagh, C.F. Carlborg, T. Haraldsson, G. Stemme and W. van der Wijngaart \\ KTH Royal Institute of Technology, Microsystem Technology Lab, Stockholm, Sweden
}

\begin{abstract}
We propose and demonstrate a novel one step method to integrate gold coated sensors with cartridges by dry covalent bonding. The cartridges are replica molded in an UV-curable off-stoichiometry thiol-ene (OSTE) polymer, featuring an excess of thiol functional groups that covalently bond to the surface of gold coated sensors upon contact. The method is demonstrated by the integration of a gold coated quartz crystal to a microfludic OSTE cartridge. The resulting bond interface is shown to be completely homogenous and void free and the package is tested successfully to a differential pressure of up to 2 bars. The performance of the biosensor chip is evaluated by measuring the unspecific binding of $0.5 \%$ albumin, resulting in a total frequency drop $205 \mathrm{~Hz}$. This approach delivers a simple but rapid high quality integration aiming for the production of robust, low cost and disposable biosensor chips.
\end{abstract}

\section{KEYWORDS}

Biosensor, quartz crystal microbalance, QCM, surface plasmon resonance, SPR, gold, thiol, off-stoichiometry thiol-ene, OSTE, integration, packaging, microfluidics.

\section{INTRODUCTION}

Biosensors are becoming well established both in research and for commercial use. By offering high sensitivity without the need for labeling, biosensors are used for a range of different biological measurements, such as the real-time study of ligand-receptor kinetics or the rapid detection of biomarkers or pathogens $[1,2]$. Two of the most common types of biosensors today are quartz crystal microbalance (QCM) and sensors based on surface plasmon resonance (SPR). QCM has gold electrodes on its quartz surface for electrical contacting and actuation while SPR based devices have a gold coated surface for supporting the surface plasmon. Both sensor technologies exist in high performance desktop lab instruments [3, 4] but they also have a great potential for use in portable instruments and point-of-care (POC) devices, especially QCM, which only requires small scale electronics to operate.

Many biosensors have a coating of gold and a range of surface chemistries have been developed aimed at the biofunctionalization of such gold surfaces. Recently, the formation of self-assembled monolayers (SAM) of alkanethiols used as linkers for attaching capturing molecules, e.g. antibodies, has become one of the most attractive methods for functionalizing gold coated biosensors [5]. Alkanethiols that are bonded via the thiol group to gold have the possibility to move around laterally on the surface, enhancing the self-assembly. Surface bound molecules in a SAM can even displace contaminants on the surface upon lateral movement, indicating that the surface diffusion effect can be very strong [6].

The great potential of using gold coated biosensors, especially quartz crystals, in low cost disposable chips for portable instruments and POC devices is often identified but very few have been realized. The lack of methods for low cost, high volume integration of the transducers in disposable cartridges limits the commercial use to desktop lab instruments using expensive sensor cartridges. Today's de-facto QCM packaging standard relies on the manual assembly of a crystal, clamped between two o-rings in a screw mounted and multi-structured cartridge. This method is incompatible with low cost, high volume fabrication and also risks decreased performance due to clamping induced stress on the sensor. One-sided adhesive attachment of the crystal to the cartridge [7] potentially allows the crystal to vibrate more freely but suffers from aging of the bond quality and the poor inertness of adhesives, leading to unspecific interactions with the sample fluid and hence limitations in the detection limit. Polydimethylsiloxane (PDMS) is a popular material for the fabrication of microfluidic packages and it can be bonded to quartz crystals by (bio-incompatible) plasma surface activation of the quartz surface [8]. However, PDMS has several drawbacks, e.g. suffering from sample absorption and monomer leaching, swelling, rapid aging of surface modifications and most importantly, it cannot offer a robust cartridge for the sensor [9]. Glue bonding can potentially be used to integrate the sensor to e.g. a thermoplastic, such as polycarbonate or an acrylic, which are better suited as the fabrication material for robust sensor cartridges than PDMS due to a higher Young's modulus. However, gluing would require an additional contact printing process step and may result in bioincompatibility and overflow of the adhesive upon applied pressure when bonding. If the QCM technology is ever going to get established for biosensing applications in the field, a clever way of integrating quartz crystals to robust, low cost and disposable polymer cartridges is needed.

We recently introduced a novel platform of multi-functional and UV-curable off-stoichiometric thiol-ene polymers, specifically designed for microfluidics and lab-on-chip applications [9]. The OSTE polymer is made from two monomers, one with thiol functional groups, $x \mathrm{R}_{1}-(\mathrm{SH})_{m}$, and the other with allyl functional groups, $y \mathrm{R}_{2}-\left(\mathrm{CH}_{2}-\mathrm{CH}=\mathrm{CH}_{2}\right)_{n}$. By tuning the backbone of the monomers, $\mathrm{R}_{1}$ and $\mathrm{R}_{2}$, the stoichiometric ratio, $x / y$ where $x \neq y$, and the number of functional groups 
on each monomer, $m$ and $n$, the OSTE polymer can adapt different material and functional properties, such as the Young's modulus (E), the glass transition temperature $\left(\mathrm{T}_{\mathrm{g}}\right)$, and the type and number of functional groups. It can be made to have a low Young's modulus for soft lithography and achieve PDMS-like properties, without suffering from the inherent drawbacks of the silicone. In contrast, its properties can also be made similar to many thermoplastics, having a high Young's modulus and being suitable both for rapid prototyping by machining and batch fabrication by molding. Furthermore, OSTE polymers feature an excess of either thiol or allyl functional groups on their surface and have the possibility to covalently bond to a range of different materials. Additionally, the OSTE material exhibits limited absorption of liquid and is easily surface modified for optimum liquid flow conditions as well as for reduced non-specific binding.

\section{NOVEL INTEGRATION APPROACH}

In this paper we propose and demonstrate, for the first time, the direct covalent binding of a gold coated sensor to a cartridge, here manufactured in OSTE. The integration approach is based on the formation of thiol-gold bonds between a thiol-excess OSTE polymer cartridge and a gold coated sensor, solely by bringing the components in contact.

By tuning the polymer to display an excess of thiol groups, i.e. $m>n$ and/or $x>y$, it offers an uncomplicated and robust integration to gold surfaces via the formation of gold-thiol bonds in a similar manner as during the formation of SAMs on such surfaces. When the materials are brought in contact, the surface groups of the polymer can covalently attach via the thiol molecules to the gold surface and then partly reorganize on the surface until optimal bond conditions are reached. This minimizes the bonding prohibiting effects of surface roughness, mechanical stress at the interface and sterical hindrance between the polymer chains reaching for the gold surface. To further enhance the bond, the bonding temperature can be increased to the glass transition temperature of the polymer to make the surface diffusion faster and the polymer softer such that it more easily conforms to surface roughness.

Two major material requirements for the biosensor cartridge are the material rigidity and the thiol surface functional groups. These properties are obtained by mixing the monomers tetra-thiol-penta-erythritol and tri-allyl-tri-azine (fig. 1) with a 2:1 stoichiometric ratio of the functional groups, which after UV-curing results in a polymer with an excess of thiol functional groups and a Young's modulus of $250 \mathrm{MPa}$ at room temperature. This OSTE polymer has a $\mathrm{Tg}=37^{\circ} \mathrm{C}$, which allows it to be softened and bonded to gold coated biosensors in a temperature range that is biocompatible. This ensures the possibility for biofunctionalization of the sensor prior to bonding without risking damage to the immobilized molecules, such as antibodies. For applications with no

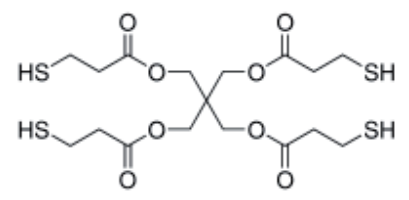

Tetra-thiol-penta-erythritol<smiles>C=CCn1c(=O)n(CC=C)c(=O)n(CC=C)c1=O</smiles>

Tri-allyl-tri-azine
Figure 1. The two monomers constituting the OSTE polymer.

requirements on low temperature bonding, a different monomer mixture could be selected to create a stiffer polymer that is softened at higher bonding temperatures to still ensure proper surface contacting during bonding

When integrating resonating biosensors, such as quartz crystals, care has to be taken to minimize the negative influences from the crystal mounting on the resonance from the crystal mounting to the cartridge. The vibration amplitude of the quartz crystal when resonating occurs at the crystal center, and declines towards the outwards edge of the crystal. The region of the crystal with non-negligible vibration is mainly defined by the region with the strongest driving electric field, i.e. where both electrodes face each other. This means that for a typical electrode configuration with a small bottom electrode and a larger concentric top electrode, the small bottom electrode determines the sensitive area of the large top electrode which is exposed to the sample liquid. When traditional mounting by double-sided clamping is used, the crystal is commonly mechanically contacted on both sides at the region of the outer perimeter of the large top gold electrode (fig. 3e). This ensures that the sample liquid is confined to the gold coated part of the crystal surface, while minimizing the negative influences on the oscillation from the mounting. In this work, this same region on the top electrode is used for mounting to the OSTE cartridge, with a single sided covalent bond to the crystal, ensuring a liquid tight seal but with potentially less mounting induced stress compared to traditional double-sided clamping.

The benefits of the novel method include that it allows for one step integration at low temperature in a dry environment, is uncomplicated and rapid, and eliminates the need for clamping, the necessity of manual assembly, the use of elastic and bioincompatible cartridge materials, or surface activation/coating.

\section{FABRICATION}

The tetra-thiol and tri-allyl monomers are mixed and poured onto a structured silicone template for replica molding (fig. 2a). Short plastic tubes are molded into the polymer cartridge to serve as fluidic inlet and outlet ports, by placing them on the silicone template before adding the monomer mixture. The material is polymerized by exposure to $365 \mathrm{~nm}$ UV-light at $50 \mathrm{~W}$ during $80 \mathrm{~s}$ (fig. 2b). After polymerization, the OSTE polymer cartridge is released from the mold template (fig. 2c). It accommodates an $8 \mathrm{~mm}$ diameter and $80 \mu \mathrm{m}$ deep hollow, that defines the microfluidic chamber on top of the quartz crystal and to which the fluidic ports are connected. 


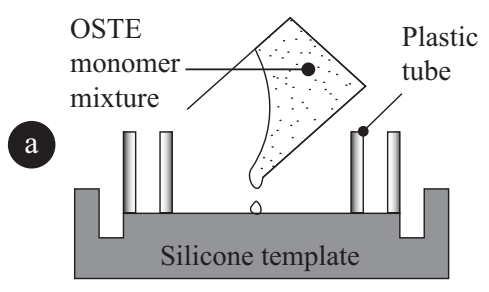

Cartridge is aligned to the crystal

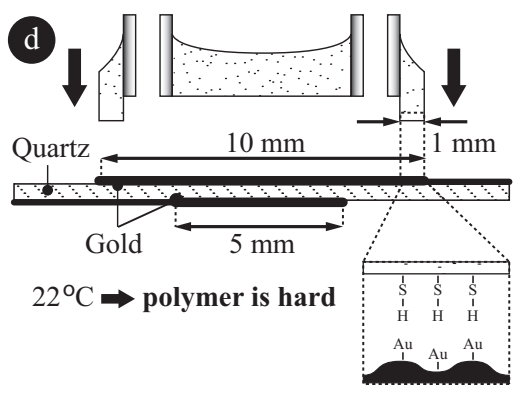

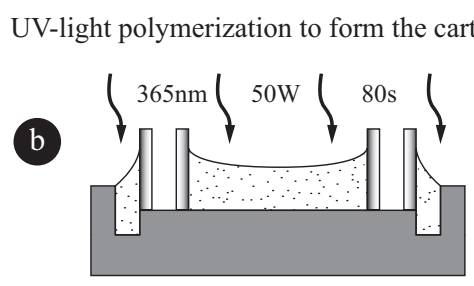

Covalent bonding of the cartridge to the crystal

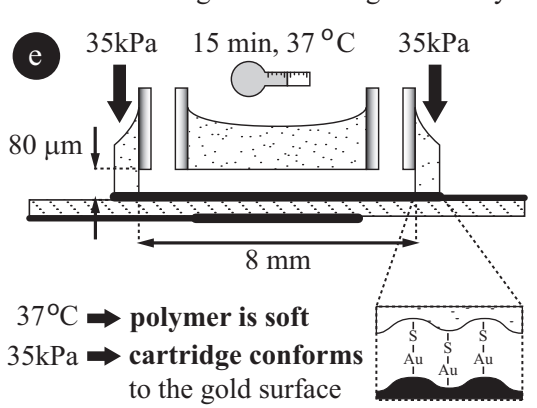

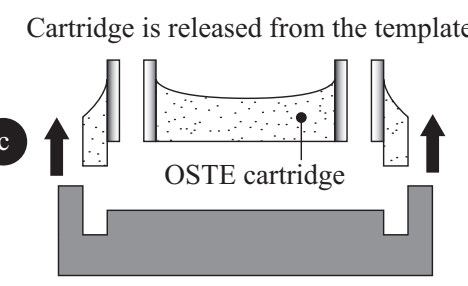

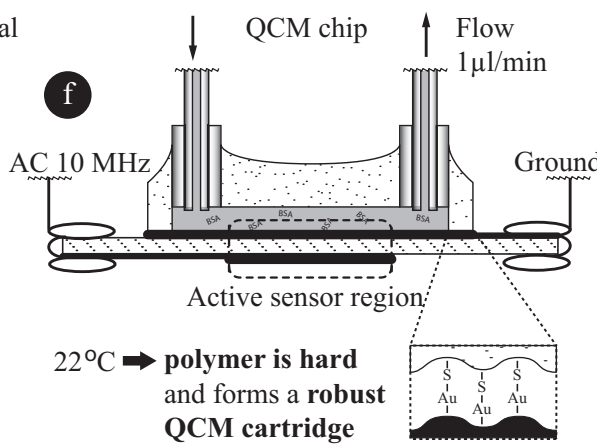

Figure 2. Fabrication process; a) replica molding of the polymer cartridge, b) polymerization by UV-light exposure, c) release of the cartridge from the template, d) alignment of the cartridge to the edge of the top gold electrode on the crystal, e) low temperature, dry bonding of the cartridge to the crystal, $f$ ) the fabricated QCM chip is connected to an oscillator and inlet/outlet ports are connected with tubing to a syringe pump.

Surrounding the hollow is an elevated $1 \mathrm{~mm}$ wide rim with a diameter of $9 \mathrm{~mm}$ that is used as the cartridge-to-crystal bond area.

A $14 \mathrm{~mm}$ diameter crystal with a $10 \mathrm{~mm}$ diameter top gold electrode and a $5 \mathrm{~mm}$ diameter bottom gold electrode (International Crystal Manufacturing Co Inc., USA) is used. The fabricated cartridge is brought in contact with the outer perimeter of the top gold electrode surface on the crystal in dry condition (fig. 2d). During bonding, pressure $(35 \mathrm{kPa})$ and heating $\left(37^{\circ} \mathrm{C}\right)$ to $\mathrm{Tg}$ are applied for 15 minutes, which softens the polymer and ensures a conformal contact at the bond interface (fig. 2e). The thiol functional groups of the OSTE polymer form covalent bonds to the gold surface, which chemically and mechanically mounts the crystal to the cartridge. As the temperature is decreased to room temperature, the polymer hardens and forms a robust cartridge for the sensor (fig. 2f). Tubes for external liquid pumping are connected to the fluidic ports and the package is mounted and electrically connected with a standard crystal holder (International Crystal Manufacturing Co Inc., USA).

\section{RESULTS}

The fabricated biosensor chip, consisting of the OSTE cartridge and the gold coated quartz crystal mounted in the crystal holder, is shown in fig. 3a. The polymer-gold bond interface is indicated with dashed lines in fig. 3b. Optical inspection of the bond interface reveals a homogenous and void free bond (fig. 3c). Filling the flow cell with Rhodamine $6 \mathrm{G}$ and measuring the fluorescence show that there is no delamination or micro cracks occurring along the bond edge (fig. $3 \mathrm{~d}$ ). The crystal and cartridge are shown side-by-side in fig. $3 e$, in which the surfaces used for bonding are indicated with white dashed lines and the outer radius of the sensitive region of the crystal used for measuring is indicated with a black dashed line. Side views of the bond interface (fig. 3f-g) indicate that the cartridge and the crystal make conformal contact along the bond edge with no visible gaps. The microfluidic chamber of the cartridge was successfully tested for leak-free operation up to 2 bars of differential pressure, by far exceeding typical biosensor operational conditions, and the OSTE polymer could support the 1:100 aspect ratio of the chamber without collapsing.

To experimentally verify the functionality of the fabricated biosensor chip, the crystal was connected to a $10 \mathrm{MHz}$ lever oscillator (International Crystal Manufacturing Co Inc., USA), and to a programmable syringe pump (Model 540060, TSE Systems GmbH, Germany). The read-out was done with a frequency counter (Model PM6666, Philips, the Netherlands) connected to a computer running a custom made Labview program (National Instruments, USA). Phosphate buffered saline (PBS, Sigma-Aldrich, USA), was pumped at $1 \mu \mathrm{l} / \mathrm{min}$ until the frequency stabilized, and $0.5 \%$ bovine serum albumin in PBS (BSA, Sigma-Aldrich, USA) was sequentially injected under the same flow conditions while the frequency was recorded (fig. 4). The first injection of BSA resulted in a $190 \mathrm{~Hz}$ frequency drop while the two following injections resulted in $10 \mathrm{~Hz}$ and 5 $\mathrm{Hz}$ frequency drops, indicating unspecific binding of BSA to the sensor surface until saturation. The noise level was recorded to $\pm 4 \mathrm{~Hz}$ at $1 \mathrm{~Hz}$ sampling frequency and no drift was visible during the measurement, demonstrating the suitability of the method for biosensor packaging.

\section{CONCLUSION}

This work proposes a novel approach for the integration of gold coated sensors with polymer cartridges. For the first time, integration by direct covalent binding between a biosensor and its cartridge is 

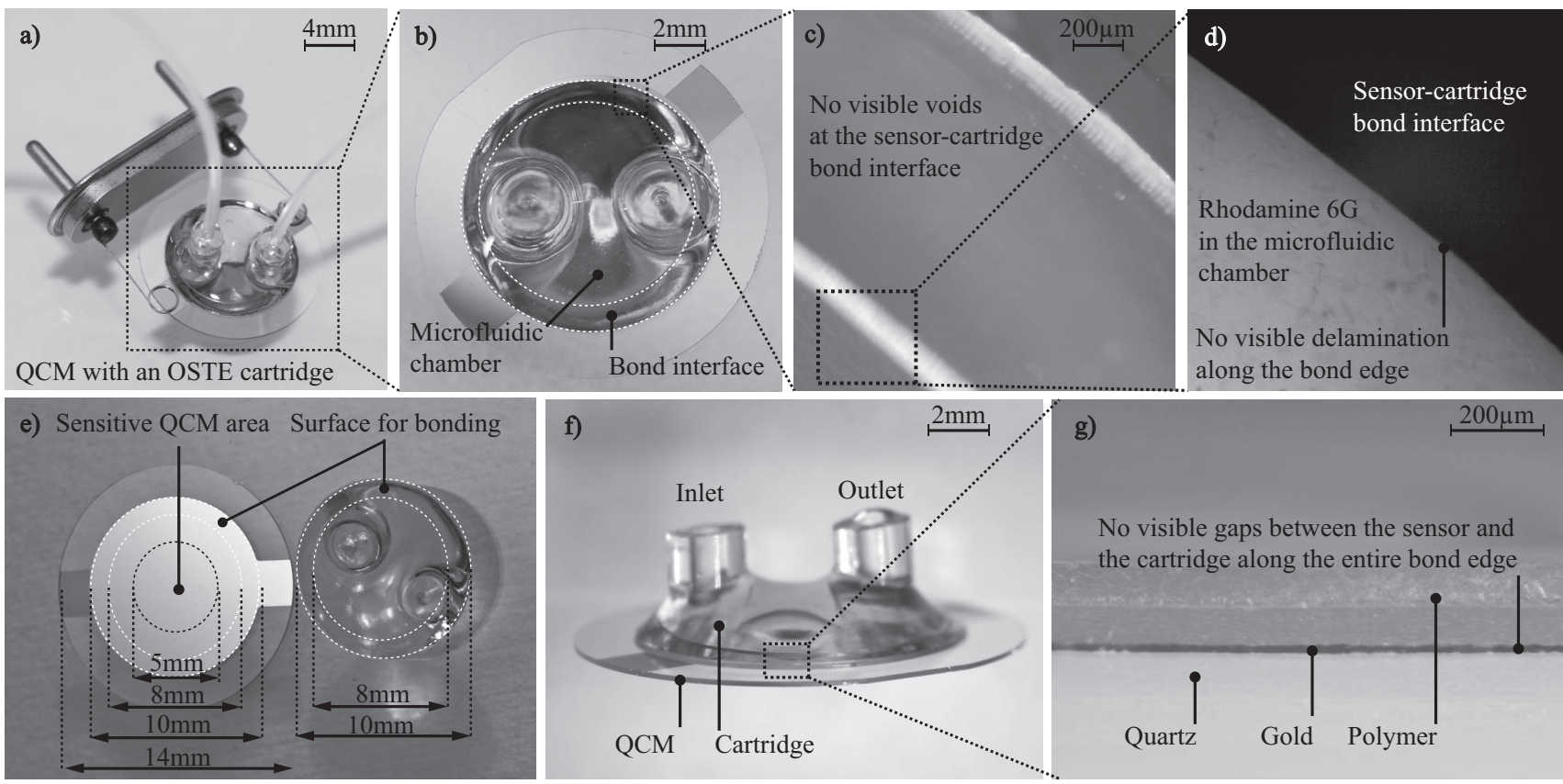

Figure 3. Fabrication results; a) a fabricated QCM chip, b) top view of the QCM chip, c) top view enlargement of the bond interface, d) fluorescent image of Rhodamine $6 G$ in the microfluidic chamber next to the bond interface, e) the top side of a crystal and the bottom side of a cartridge, f) side view of the QCM chip, g) side view enlargement of the bond interface.

demonstrated. The cartridge is made of a newly developed OSTE polymer which has an excess of thiol functional groups, allowing it to covalently bond to the gold surface of the sensor. The OSTE polymer features several advantages compared to commonly used microfludic cartridge materials, such as PDMS and thermoplastics, and has a promising potential for use in robust, low cost and disposable biosensor chips.

The fabrication method of the OSTE cartridges is based on replica molding with rapid UV-curing and is therefore applicable to microstructured components and scalable up to high volume fabrication. The integration of the gold coated sensor with the OSTE cartridge is done in a one step process by biocompatible dry bonding where covalent bonds are formed when bringing the two components together. Mild heat and pressure are applied during bonding, but only to further enhance the bond

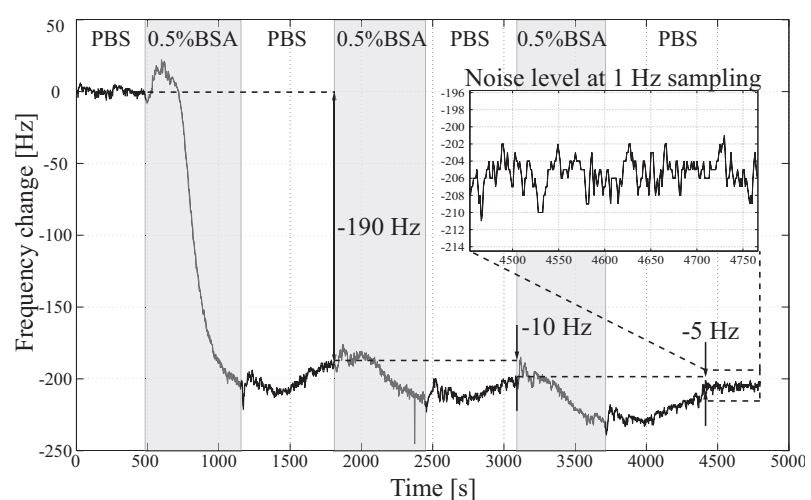

Figure 4. Sensorgram for a QCM chip. PBS and $0.5 \%$ BSA was sequentially injected, indicated as white and grey areas, respectively. The resulting shifts were $-190 \mathrm{~Hz},-10 \mathrm{~Hz}$ and 5 $\mathrm{Hz}$, indicating saturation of the sensor surface. The inset shows the noise level at $1 \mathrm{~Hz}$ sampling frequency. quality. The process addresses the need for a simple and straightforward production of robust, low cost and disposable sensor chips and eliminates many of the limitations of previous methods.

This proof-of-concept demonstrates the great potential of the OSTE polymer as a biosensor cartridge material and its simple integration to gold coated sensors. Future work will extend the concept from the minimalistic design of the microfluidic cartridge to a complete biosensor chip which fully encloses the sensor.

\section{ACKNOWLEDGEMENT}

This work was sponsored by the Swedish Governmental Agency for Innovation Systems, the Swedish Foundation for Strategic Research, and the Swedish Research Council.

\section{REFERENCES}

[1] Ferreira et al., Trends in Biotechnology, 27(12), 689-697, 2009

[2] Homola, Chemical Reviews, 108 (2), 462-493, 2008

[3] www.attana.se, Attana AB, 20110325

[4] www.biacore.se, GE Healthcare, 20110325

[5] Frasconi et al., Analytical and Bioanalytical Chemistry, 398(4), $1545-1564,2010$

[6] Troughton et al., Langmuir, 4 (2), 365-385, 1988

[7] Frisk et al., Lab-chip, 8, 1648-1657, 2008

[8] Michalzik et al., Sensors and Actuators B: Chemical, 111-112, 410-415, 2005

[9] Carlborg et al., $\mu$ TAS 2010, Groningen, 70-72, 2010

\section{CONTACT}

*N. Sandström, tel: +46-8-790-9231;

niklas.sandstrom@ee.kth.se 